Patricia Whitelock and Russell Cannon, eds.

\title{
ROSAT HRI and PSPC Observations of M32
}

\author{
Z. Zang and E.J.A. Meurs \\ Dunsink Observatory, Castleknock, Dublin 15, Ireland
}

\begin{abstract}
The X-ray emission from M32 has been studied with ROSAT PSPC and HRI observations. A new analysis of the best available exposure with M32 in the central part of a PSPC frame improves on a previously published analysis and indicates only one source associated with M32. Our analysis of the dedicated HRI exposure of M32 also leads to different conclusions than published recently. At HRI resolution M32 appears as an extended source, probably a little offset from the optical nucleus. Possible interpretations are discussed, which are relevant to the question whether a low-level active nucleus may be present or not.
\end{abstract}

\section{Introduction}

M32 (NGC 221) is one of the nearest elliptical galaxies. The optical imaging and spectroscopy indicate that M32 contains a central dark object, probably a black hole, of mass $\mathrm{M} \cong 3 \times 10^{6} M_{\odot}$ (Bender et al. 1996; van der Marel et al. 1997). The Einstein satellite detected one source with a total $0.2-4 \mathrm{keV}$ luminosity of $\mathrm{L}_{X} \sim 5.4 \times 10^{37} \mathrm{erg} \mathrm{s}^{-1}$ (Fabbiano 1989), and HRI position (J2000) $\mathrm{RA}=$ $00^{\mathrm{h}} 42^{\mathrm{m}} 42.4^{\mathrm{s}}$, Dec $=40^{\circ} 51^{\prime} 55^{\prime \prime}$ (Crampton et al. 1984).

Since it is a satellite galaxy of M31, M32 is included in 4 out of 6 pointed observations of the first ROSAT PSPC survey of M31 which was carried out during July 1991 (Supper et al. 1997). The best PSPC exposure (ID. 600068) combines an exposure time of about $30 \mathrm{ks}$ with a position of M32 in the inner detector area. An analysis of these data by Eskridge et al. (1996) suggests a weaker source just NE of the main contributing source, demonstrates several spectral shapes to fit the data and summarizes the evidence for either a low-level nuclear source or possibly several sources of stellar origin.

The HRI observation which is centred at M32 was recently published (Loewenstein et al. 1998). M32 is associated with one fairly strong source some $7^{\prime \prime}$ away from the optical centre of the galaxy. There is a suggestion that after subtracting this source some residual emission remains closer to the centre of M32.

\section{New Analysis of ROSAT PSPC and HRI Data}

\subsection{Morphology}

Our X-ray image of the best available PSPC observation with ID. 600068 benefits from a re-processing of the satellite data and shows no evidence for any 


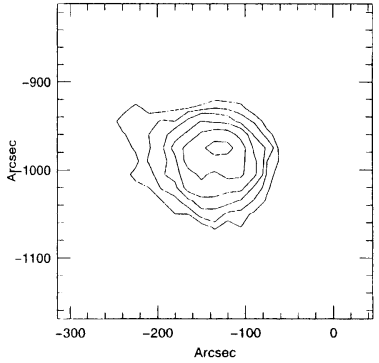

(a)

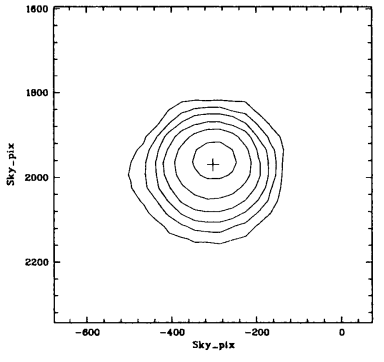

(b)

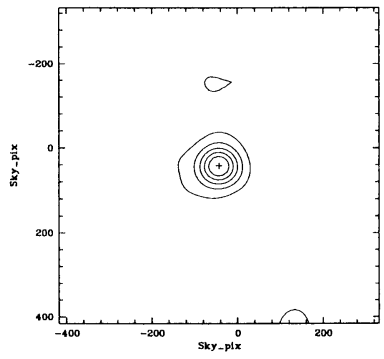

(c)

Figure 1. Contour plots of the X-ray source associated with M32, in a $6.25^{\prime} \times 6.25^{\prime}$ field: (a) PSPC analysis of Eskridge et al. (1996), (b) our PSPC result for the same field, and (c) our HRI contours. Notice that $1^{\prime \prime}$ equals 2 sky-pixels. The cross in (b) and (c) refers to the position found for the X-ray source.

additional source located just NE of M32. The previous result of that kind (Eskridge et al. 1996) may be due to some bad satellite attitude solution. The HRI observation does not detect any source at that location either. Figure 1 presents the contours of the PSPC and HRI observations of M32.

Our analysis of the M32 HRI field results in a source for which the source detection algorithm returns a position that agrees closely with the brightness peak (Fig. 1c). Loewenstein et al. (1998) however found a source position that is shifted by about $5^{\prime \prime}$ towards the NW. Since after a source subtraction exercise some residual emission is left closer to the centre of M32 where also their source detection algorithm finds a weak second source, they hint at the possibility that a weak X-ray source in the nucleus of M32 pulls the detection position towards it. In Section 3.1 we show that this is an unlikely interpretation, supporting the correctness of our plotted detection result.

The source detection routine attributes a small extension to the source associated with M32. To investigate this further we determined the radial brightness profile (Fig. 2a) and the azimuthal brightness variation (Fig. 2b) for this source. The azimuthal variation shows a fairly symmetric double wave with peaks of comparable amplitude, as may be expected for an elongated source.

\subsection{Spectrum}

We have determined the X-ray spectrum for the reprocessed PSPC data. The contribution from the background was determined in an annulus around the source. There is no great change in our results compared to Eskridge et al. (who combined photons from three long observations), except for the fact that they used too many spectral bins for their fits (more than 100) while we with 21 stay closer to an optimum spectral binning for PSPC data (cf. Davelaar 1969). 


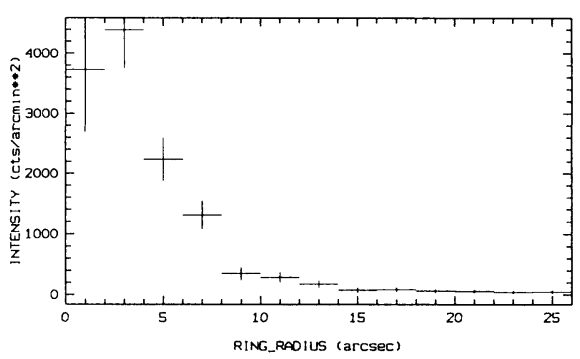

(a)

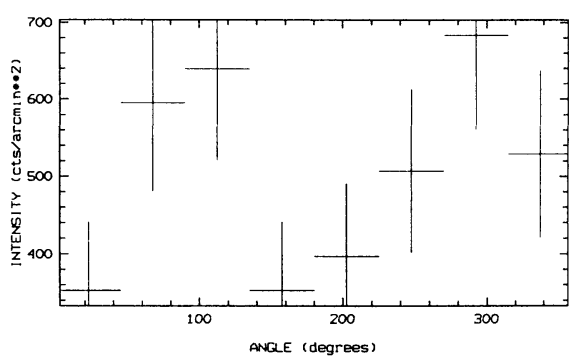

(b)

Figure 2. M32 ROSAT HRI brightness profiles within 26": (a) radial profile, (b) azimuthal variation, angle starts from North clockwise.

\subsection{X-ray Position vs Optical Positions}

A few of the (non-M32) HRI sources have optical counterparts that suggest positional corrections of $-2^{\prime \prime}$ in RA and $+3^{\prime \prime}$ in declination. When these adjustments are applied, the offset of the X-ray source to the optical nucleus of M32 is still about $6^{\prime \prime}$, roughly in Easterly direction. This refers to the most accurate optical position $\left( \pm 0.7^{\prime \prime}\right)$ listed in the NED, but these measurements could have incurred greater errors for objects like M32. Other optical positions in NED with errors $\pm 4^{\prime \prime}$ yield offsets of about $3^{\prime \prime}-6^{\prime \prime}$. M32 may require a dedicated optical position measurement before the X-ray/optical offset can be reliably established.

\section{Discussion}

\subsection{Extended or Multiple Source}

The idea that a source at a few arcsec distance could cause a shift in detection position (see Section 2.1) as reported by Loewenstein et al. (1998) can be tested with a simple simulation. Two HRI sources are generated (within EXSAS) and added together at $10^{\prime \prime}$ separation with count ratios varying between 12:12 and 12:1 (the latter roughly the ratio quoted in Loewenstein et al.). The effect of the second source on the position returned by the detection algorithm is that, as expected, a halfway position is found for two equal sources, but for the ratio relevant for the case of M32 any shift is less than $1^{\prime \prime}$. With our own detection result coinciding with the peak of the brightness distribution, we conclude that the position offset in Loewenstein et al. is probably not correct. This casts doubt on their source subtraction attempt and we prefer source extension above a putative additional source, which is supported by the clear azimuthal variation in Fig. 2b.

\subsection{PSPC and HRI Fluxes}

Loewenstein et al. (1998) have summarized the X-ray brightness history of the M32 source. The PSPC data points are often above the HRI flux, but some are comparable. This implies real increases in brightness rather than the PSPC 
resolution allowing a completer flux measurement of an extended source. Over the longest time span available, from Einstein till ASCA, there seems to be a base level for the M32 flux and only the ROSAT PSPC appears to have witnessed a flaring interval. Perhaps the base level indicates the X-ray flux from an extended component, in which the source of the X-ray variation is situated.

\subsection{Nature of the M32 Source}

The present study shows the X-ray source associated with M32 as one source, but probably with intrinsic structure. The possible source extension and the uncertain offset from the optical nucleus bear on the interpretation of this source.

The source extension would be an important clue. Depending on the possible positional consistency with the centre of M32, the source could be a low-level active nucleus, a SNR or perhaps a star-formation complex. The last possibility is not likely since no on-going star formation is known for M32, unless the source is a chance superposition and belongs to M31. Another possibility - which may increase the likelihood that a source appears extended - is a chance superposition of a Galactic source. If the source is a nuclear source in M32, then an interesting interpretation for the flaring observed with PSPC (see section 3.2) is that it could have been a stellar disruption flare as described by Rees (1988). Perhaps such a flare is superposed on a small specimen of hot gas halo as often observed around $\mathrm{E}$ and S0 galaxies.

The current status of positional information in the optical would favour a SNR interpretation. A size of around $10^{\prime \prime}$ corresponds to just over $30 \mathrm{pc}$ at the distance of M32, which is rather normal for SNRs in our galaxy and in the LMC. The observed brightness increases during the PSPC exposures could originate from an associated pulsar. Notice that Eskridge et al. (1996) found a weak indication for a period of 1.27 days. This is slow for a pulsar but it could be part of a binary that survived the supernova explosion. Its age could be consistent with the intermediate population that several authors have noticed from optical spectroscopy. Given the expected lifetimes involved, the supernova itself would have been of type Ia, which is indeed typical of spheroidal objects including bulges. The observed X-ray luminosity is consistent with such a LMXB, together with possibly a SNR.

\section{References}

Bender, R., Kormendy, J., Dehnen, W. 1996, ApJ, 464, L123

Crampton, D., Cowley, A.P., Hutchings, J.B., et al. 1984, ApJ, 284, 663

Davelaar, J. 1969, Thesis, University of Leiden

Eskridge, P.B., White, R.E., Davis, D.S. 1996, ApJ, 463, L59

Fabbiano, G. 1989, ARA\&A, 27, 87

Loewenstein, M., Hayashida, K., Toneri, T., Davis, D.S. 1998, ApJ, 487, 681

Rees, M.J. 1988, Nature, 333, 523

Supper, R., Hasinger, G., Pietsch, W., et al. 1997, A\&A, 317, 328

van der Marel, R.P., de Zeeuw, P.T., Rix, H.-W. 1997, ApJ, 488, 119 